\title{
NEUROGENIC MODULATION OF MICTURITION: THE RELATION BETWEEN STIMULATION INTENSITY AND THE MAXIMUM SHORTENING VELOCITY OF THE GUINEA PIG DETRUSOR MUSCLE
}

\author{
J. GROEN,* R. VAN MASTRIGT AND R. BOSCH \\ From the Department of Urology, Erasmus University Rotterdam, Rotterdam, The Netherlands
}

\begin{abstract}
The course of micturition depends on bladder contractility and urethral resistance. The former is determined by geometrical, muscular and neurogenic factors. The muscular aspects of bladder contractility can be characterized by the parameters $\mathrm{P}_{\mathrm{isv}}$, the isovolumetric detrusor pressure, and $v_{\max }$, the maximum (unloaded) shortening velocity of the detrusor muscle. The neurogenic control system of the urinary tract modulates bladder contractility, which might effectively change the values of $P_{\mathrm{isv}}$ and $v_{\max }$. These parameters also depend on the instantaneous bladder volume. In previous work the dependence of $P_{\text {isv }}$ on the intensity of stimulation and bladder volume was measured in guinea pig bladders in vivo and in vitro. In the present work $v_{\max }$ was derived in 5 guinea pig bladders in vitro, using electrical stimulation and the stop-flow technique. This technique implies that pressure values measured at a certain shortening velocity of the bladder circumference and in an isovolumetric contraction at the same volume are used to derive $v_{\max }$ mathematically from the Hill equation. $v_{\max }$ was independent of the bladder volume in the range of 0.6 to $6.1 \mathrm{ml}$., but it was significantly different for the two intensities of stimulation used. Therefore, it is concluded that the maximum shortening velocity of the guinea pig detrusor muscle depends on the intensity of stimulation. During submaximal stimulation the detrusor not only generates lower pressures, it also contracts more slowly. A possible explanation for this phenomenon is that the bladder is not uniformly stimulated.

The isovolumetric pressure measured in the stop-flow test was compared with the isovolumetric pressure measured at the same bladder volume some minutes later. It was observed that shortening had a depressant effect of approximately $33 \%$ on the isovolumetric pressure. This implies that the clinically employed stop-flow test might underestimate detrusor contraction strength.
\end{abstract}

KEY WORDS: bladder, guinea pigs, urodynamics, muscle contraction

The course of micturition depends on the contraction strength of the bladder and the resistance offered to urinary flow by the urethra. The contractile properties of the bladder are determined by factors related to the detrusor muscle and the nervous system. Inadequate or insufficiently sustained neurogenic stimulation of the detrusor muscle is therefore one of the possible causes of inefficient voiding, causing residual urine. In a series of experiments we studied the modulation of bladder contractility by neurogenic factors. ${ }^{1-3}$

The muscular aspect of bladder contractility is defined by the relationship between two variables: the shortening velocity $\mathrm{v}$ of the bladder circumference and the active detrusor pressure $P(v)$ at that shortening velocity. This relationship can be modelled by a hyperbolic equation: ${ }^{4}$

$$
\left[\mathrm{P}(\mathrm{v}) / \mathrm{P}_{\text {isv }}+0.25\right] \times\left[4 \times \mathrm{v}+\mathrm{v}_{\max }\right]=1.25 \times \mathrm{v}_{\max }
$$

where $\mathrm{P}_{\mathrm{iav}}$ is the active detrusor pressure in an isovolumetric contraction and $v_{\max }$ is the bladder circumference shortening velocity at zero load (fig. 1). Active pressure is defined as the pressure exceeding the (passive) pressure resulting from the viscoelastic properties of the bladder wall.

According to equation (1), the contractility of a particular bladder can be fully characterized by the parameters $P_{\text {isv }}$ and $v_{\max }$. The neurogenic control system of the urinary tract modulates bladder contractility, which might effectively change $P_{i s v}$ and $v_{\max }$. Furthermore, these parameters depend

Accepted for publication July 21, 1994.

* Requests for reprints: Department of Urology, Room EE 1630, Erasmus University Rotterdam, P.O. Box 1738, 3000 DR Rotterdam, The Netherlands.

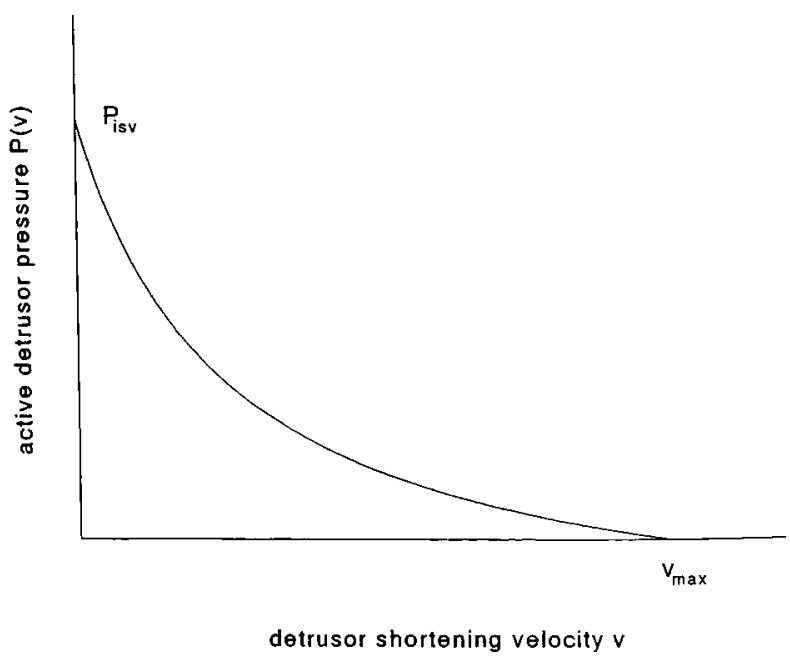

FIG. 1. Theoretical relationship between active detrusor pressure and detrusor shortening velocity. According to equation (1), this relationship can be completely characterized by intercepts $P_{\text {isv }}$ and $v_{\max }$ on both axes.

on the instantaneous bladder volume. In previous work $\mathrm{P}_{\mathrm{isv}}$ and $v_{\max }$ were estimated in guinea pig bladders in vivo, while $P_{\text {isv }}$ was also measured in vitro. ${ }^{2,3}$ It was concluded that in vivo the bladder was not fully stimulated at normal capacity: 
the isovolumetric detrusor pressure was significantly lower than that attained in vitro under conditions of optimal electrical stimulation. Unlike the in vitro pressure values, pressure in vivo did not depend on bladder volume. Also the parameter $v_{\max }$ did not depend on bladder volume in vivo. In the present study the dependence of $v_{\max }$ on the intensity of electrical stimulation and the bladder volume was studied in vitro.

\section{MATERIALS AND METHODS}

Five male guinea pigs weighing 895 to 1175 g. were anesthetized with urethane (1.2 g./kg. intraperitoneally). The bladder was exposed via a midline incision in the abdominal wall. After ligation of both ureters the bladder was excised and kept at room temperature in a buffer solution of the following composition: $\mathrm{NaCl} 137 ; \mathrm{KCl} 2.7 ; \mathrm{CaCl}_{2} 1.8 ; \mathrm{MgCl}_{2}$ $1.0 ; \mathrm{NaHCO}_{3}$ 8.0; HEPES ( $\mathrm{N}$-[2-hydroxyethyl]piperazine- $\mathrm{N}^{\prime}$ [2-ethanesulfonic acid]) 10.5 and glucose $5.1 \mathrm{mmol} . / \mathrm{l} ; \mathrm{NaOH}$ was added to adjust the $\mathrm{pH}$ to 7.4. Two catheters were inserted via the remaining part of the urethra: one $5 \mathrm{~F}$ catheter, which was connected to a disposable pressure transducer, and a stainless steel tube (i.d. $1.7 \mathrm{~mm}$.), which was connected to a Harvard infusion pump via a low-compliance plastic tube. The bladder was suspended in an organ bath containing $85 \mathrm{ml}$. of the above buffer solution, which was equilibrated with $95 \% \mathrm{O}_{2}-5 \% \mathrm{CO}_{2}$ at a temperature of $37 \mathrm{C}$ The $\mathrm{pH}$ was in the range 7.3 to 7.4 , and the osmolality was close to $300 \mathrm{mOsm}$. The solution could be refreshed within a few seconds from a heated and aerated storage container; this was done every 30 minutes. The bladder was initially filled with room temperature saline to a volume of $3 \mathrm{ml}$. to accommodate for 1 hour before the start of the experiments.

Next, the bladder volume was changed to a start value in the range of 1.9 to $7.9 \mathrm{ml}$. according to a scheme explained below. The bladder was stimulated electrically for 5.2 to 6.5 seconds at intervals of at least 5 minutes, using 2 parallel platinum electrodes $(20 \times 30 \mathrm{~mm}$.), one on each side of the bladder. Rectangular pulses were used. The applied voltage was $10 \mathrm{~V}$, while the pulse frequencies used were 25 and 200 $\mathrm{Hz}$. The pulse width was adapted to the frequency to attain a duty cycle of $50 \%$. To avoid electrolysis of the buffer solution, biphasic pulse stimulation was used. Each measurement consisted of the following phases (fig. 2): During the first 2 seconds of stimulation the bladder contracted isovolumetrically at the start volume. During the following 1.2 to 2.5 seconds the bladder was emptied using the infusion pump at

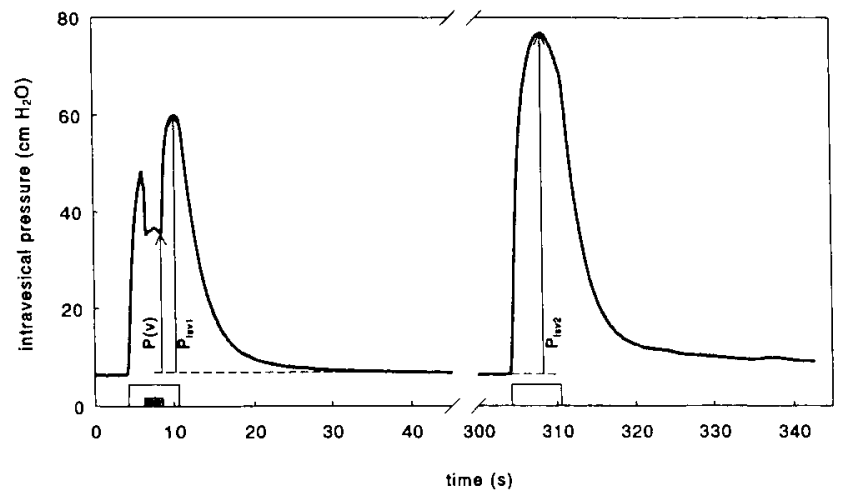

FIG. 2. Schematic representation of experimental procedure and measured pressure signal in 2 consecutive contractions in response to electrical stimulation. Stop-flow contraction was followed by completely isovolumetric contraction. During time interval indicated with blank boxes, bladder was stimulated electrically, while during interval indicated with shaded box bladder was emptied using pump. $\mathbf{P}(v)$ is active detrusor pressure at moment bladder emptying by pump was stopped; $P_{\text {iavi }}$ is maximum active pressure in isovolumetric contraction following detrusor shortening; $\mathbf{P}_{\mathrm{isv2} 2}$ is maximum active pressure in second, completely isovolumetric contraction. an imposed flow rate. During the last 2 seconds the bladder contracted isovolumetrically again at the resulting smaller volume. These three successive phases composed what will be called a stop-flow contraction. At least 5 minutes later an isovolumetric bladder contraction was induced once more by stimulating for 6.5 seconds at the last volume. All contractions were sampled at a rate of $10 \mathrm{~Hz}$ with a personal computer and stored for further analysis.

Two kinds of test measurements were performed to exclude artifacts. First the catheters were positioned at the bottom of the organ bath, which was subsequently emptied by the pump. The measured pressure was related to the volume of fluid displaced by the pump. In the second type of test measurements, the bladder experiments were repeated with the bladder replaced by a rubber balloon.

Bladder emptying during the contraction simulated micturition. Pressure was measured as a function of the continuously varying detrusor muscle length, or bladder circumference shortening velocity. This shortening velocity $v$ was calculated from the instantaneous volume and the flow rate $\mathrm{Q}$, considering the bladder a thin-walled sphere: $v=Q / 2 R^{2}$, where $R$ is the bladder radius. ${ }^{5}$ The settings used for $Q$ on the infusion pump were $0.36,0.71,1.43$ and $3.51 \mathrm{ml}$. per second. The bladder start volumes and the duration of the bladder emptying phase, which ranged from 1.9 to $7.9 \mathrm{ml}$. and from 1.2 to 2.5 seconds, were adapted to $\mathrm{Q}$ to end bladder emptying at volumes ranging from 0.6 to $6.1 \mathrm{ml}$. The $\mathrm{v}$ values thus obtained ranged from 0.23 to $2.61 \mathrm{~cm}$. per second. The experimental procedure resulted in two active pressure values at the same volume: a value $P(v)$ at the shortening velocity $v$ caused by the pump and an isovolumetric value $\mathbf{P}_{\text {isv1 }}$ (fig. 2). By dividing these two pressure values, data were obtained which were used to calculate $v_{\max }$ from equation (1). To study the volume dependence of $v_{\max }$, this parameter was derived algebraically from equation (1) for each separate measurement. Next, equation (1) was fitted to the pooled pressure ratio/shortening velocity data for each bladder and for both stimulation frequencies, where $v_{\max }$ was the parameter to be changed. The Levenberg-Marquardt algorithm was used. The resulting $v_{\max }$ values found for both stimulation frequencies were compared using the paired t test.

Because it was found that (as in fig. 2) the second isovolumetric contraction was generally much higher than the isovolumetric part of the stop-flow contraction, the ratio $\mathrm{P}_{\text {isv1 }} /$ $P_{\text {isv2 }}$ and its volume and shortening velocity dependence were also studied.

The pressure decay in the isovolumetric contractions (second part of fig. 2) was studied by dividing the active pressure $P(6.5)$ at the end of the 6.5 second stimulation period by the maximum active pressure $P_{\text {isv2 }}$ attained in this period and plotting this ratio as a function of the bladder circumference. The bladder circumference (length) dependence of the ratio $\mathrm{P}(6.5) / \mathrm{P}_{\text {isv2 }}$ was compared for both stimulation frequencies used.

In the above derivations, active pressure in the stop-flow contractions was defined as the instantaneous pressure minus the pressure after the contraction had subsided. In the completely isovolumetric contractions it was defined as the instantaneous pressure minus the pressure before the contraction.

\section{RESULTS}

Examples of the test measurements in an empty organ bath and in a rubber balloon, performed at the highest flowrate used in the bladder experiments, are shown in figure 3 . It can be seen that the measured pressure faithfully reflected the pump volume and remained constant as soon as the pump stopped. No overshoot or bias during and after pumping was observed. Figure 2 shows a typical example of the measured intravesical pressure in two consecutive contrac- 

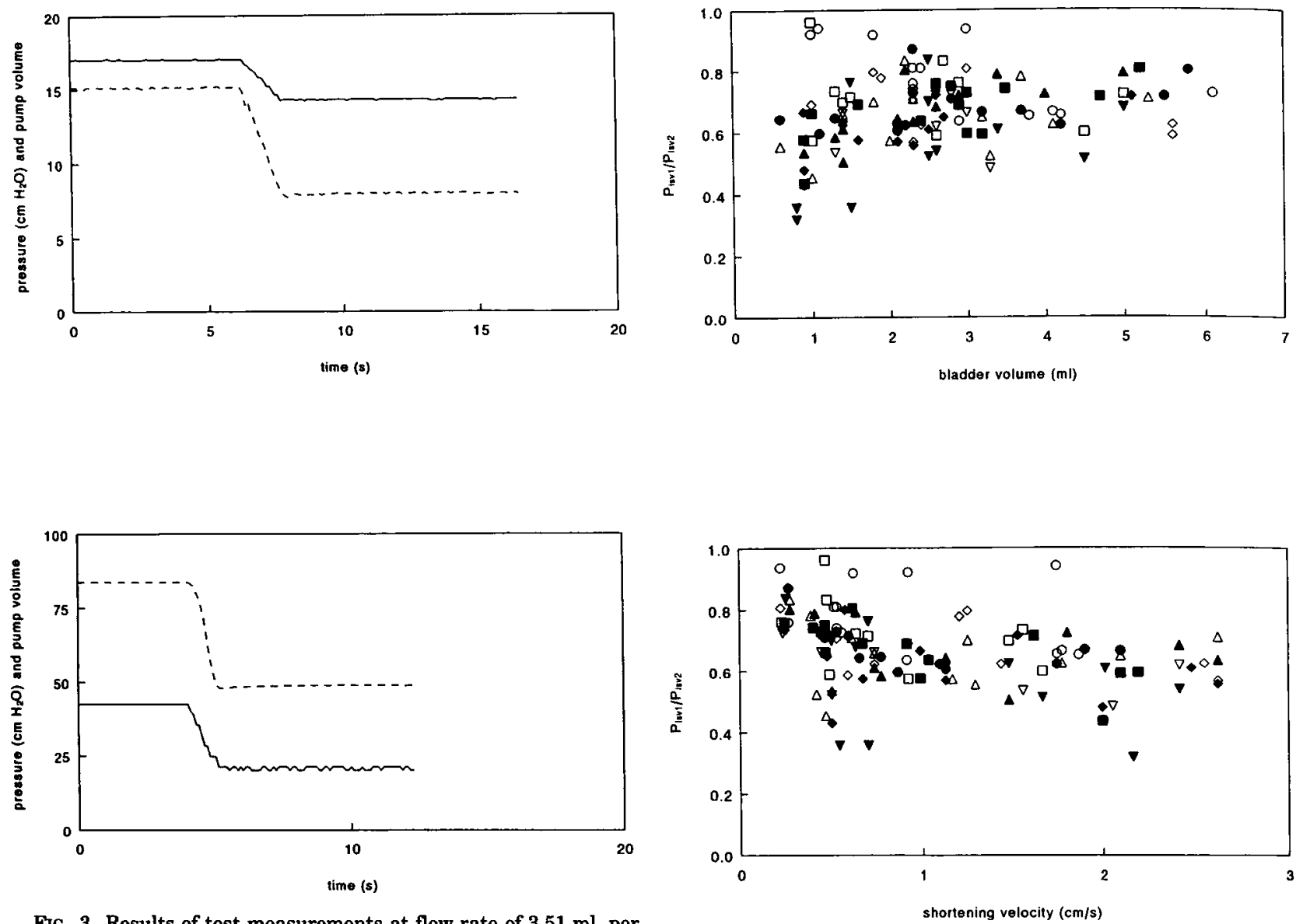

Fig. 3. Results of test measurements at flow-rate of $3.51 \mathrm{ml}$. per second. $A$, catheters were at bottom of organ bath. $B$, catheters were in rubber balloon, situated in bath. Solid lines: pump volume (arbitrary units). Broken lines: measured pressure.

tions, a stop-flow contraction with bladder emptying caused by the pump and a completely isovolumetric contraction. The effect of the flow-rate on the measured pressure is obvious. The maximum pressure values in the two consecutive contractions at the same volume were generally considerably different, as in figure 2: the mean value of the ratio $\mathrm{P}_{\text {isv } 1} / \mathrm{P}_{\text {isve }}$ of all 114 measurements (fig. 4) was $0.67 \pm 0.12$ (the standard error of the mean). For all 5 bladders and for both stimulation frequencies, the regression coefficient of this ratio on bladder volume and on shortening velocity was calculated. The 95\% confidence interval of the regression coefficient on volume contained the value $0 \mathrm{ml}^{-1}$ in 6 of the 10 cases; it contained only positive values in 3 cases and only negative values in 1 case. The regression coefficient on shortening velocity was negative in all 10 cases, but in only one case was it significantly different from $0(\mathrm{~cm} . / \mathrm{sec} .)^{-1}$ at the 0.05 level. Figure 5 shows the algebraically derived $v_{\max }$ values as a function of volume for all 5 bladders and for both stimulation frequencies. Linear regression analysis showed that in only one of the 10 cases was the regression coefficient significantly different from $0 \mathrm{~cm} . / \mathrm{sec} . / \mathrm{ml}$. at the 0.05 level. The result of fitting equation (1) to the pressure ratio/shortening velocity data of one bladder is shown in figure 6 . The five $v_{\max }$ values for the five different bladders, measured at stimulation frequencies 200 and $25 \mathrm{~Hz}$, averaged $3.75 \pm 0.08$ (standard error of the mean) and $3.00 \pm 0.22 \mathrm{~cm} . / \mathrm{sec}$. These values were significantly different $(p=0.015)$.

In the second contraction in figure 2 the pressure already started decreasing during stimulation. The decay in pressure

FIG. 4. Ratio $\mathrm{P}_{\mathrm{isv1}} / \mathrm{P}_{\mathrm{isv} 2}$ of maximum active pressure in isovolumetric contraction immediately following detrusor shortening and in completely isovolumetric contraction some minutes later as function of bladder volume $(A)$ or detrusor shortening velocity $(B)$. Each symbol represents results from 1 bladder. Filled and open symbols represent stimulation frequencies 200 and $25 \mathrm{~Hz}$.

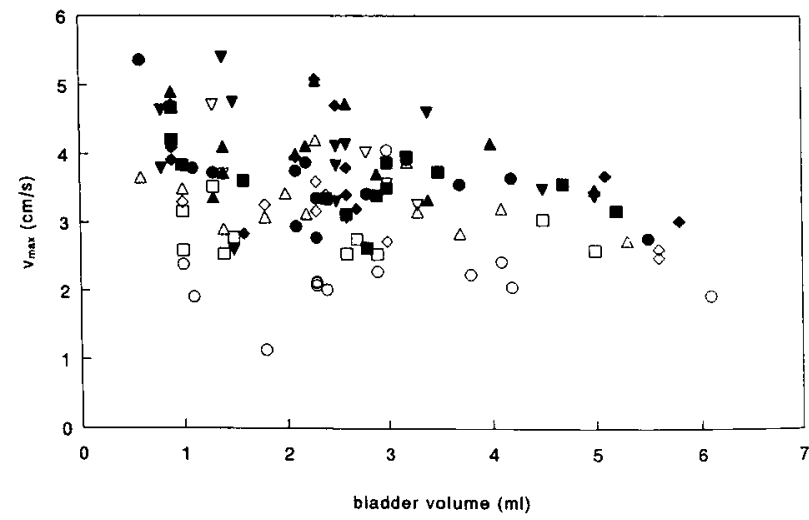

FIG. 5. $v_{\max }$ values as function of bladder volume, as derived algebraically from equation (1), for all measurements in all 5 bladders at both stimulation frequencies. Different symbols represent results from different bladders. Filled and open symbols represent stimulation frequencies 200 and $25 \mathrm{~Hz}$.

was clearly volume dependent (fig. 7, A). A straight line was fitted to all consecutive points where $\mathrm{P}(6.5) / \mathrm{P}_{\text {isv2 } 2}$ was less than 0.95 . The bladder circumference corresponding to the intersection with the line $\mathrm{P}(6.5) / \mathrm{P}_{\text {isv2 }}=1$ was called cut-off 


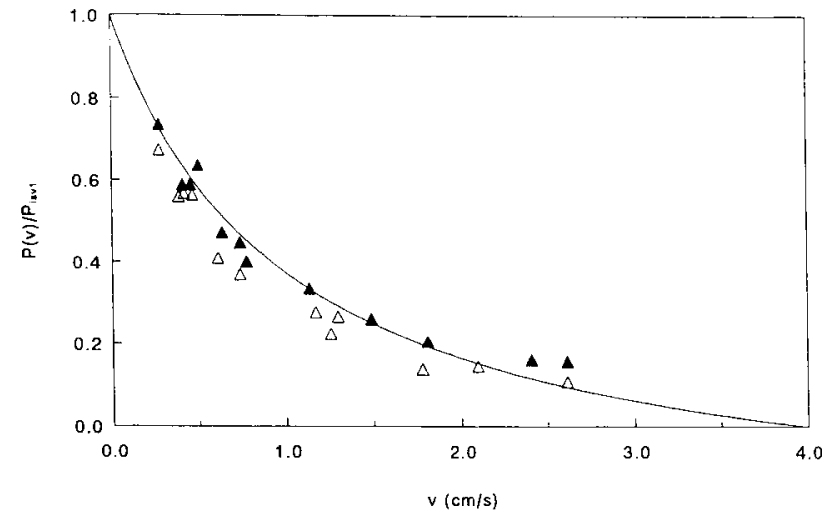

FIG. 6. Pressure ratio/shortening velocity data, obtained in 1 bladder at both stimulation frequencies: $200 \mathrm{~Hz}$ (filled symbols) and 25 $\mathrm{Hz}$ (open symbols). Curve is result of fitting equation (1) to $200 \mathrm{~Hz}$ data.
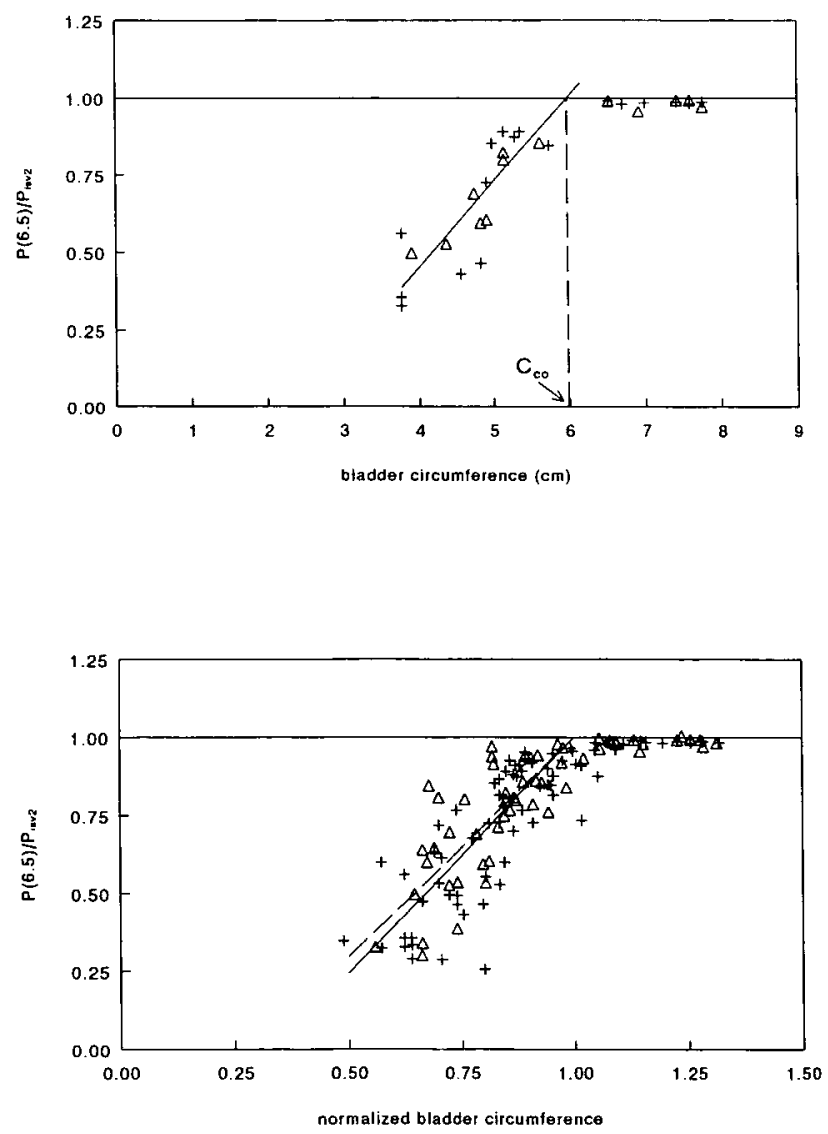

FIG. 7. $A$, pressure decay versus bladder circumference as measured in 1 bladder at stimulation frequencies $200 \mathrm{~Hz}(+)$ and $25 \mathrm{~Hz}$ $(\triangle)$. Straight line was fitted to data for which $\mathrm{P}(6.5) / \mathbf{P}_{\text {isve }}<0.95$ at $200 \mathrm{~Hz}$ stimulation. $\mathrm{C}_{\text {co }}$ is cut-off bladder circumference. $B$, pooled data of pressure decay versus bladder circumference, latter being normalized for cut-off circumference. Symbols as in $A$. Solid and dotted lines correspond to 200 and $25 \mathrm{~Hz}$ data.

circumference. It could be concluded that the cut-off circumferences at stimulation frequencies of 200 and $25 \mathrm{~Hz}$ were not significantly different ( $p=0.62$ ) and averaged to a value corresponding to a bladder volume of about $3.5 \mathrm{ml}$. In this small sample the cut-off circumference did not correlate with bladder weight ( $p=0.58$ ). Figure $7, B$ shows a plot of all $\mathrm{P}(6.5) / \mathrm{P}_{\text {isv2 }}$ values in the 5 bladders as a function of bladder circumference, normalized for the cut-off circumference. The bladder circumference dependence of the ratio $\mathrm{P}(6.5) / \mathrm{P}_{\text {isv2 }}$ was not significantly different for the two stimulation frequencies.

\section{DISCUSSION}

Maximum shortening velocity. Values for $\mathrm{v}_{\max }$, the maximum (unloaded) shortening velocity of the detrusor muscle, were determined in vitro by simulating stop-flow tests. At a number of different flow rates or shortening velocity values, two pressure values were obtained at exactly the same bladder volume. The first of these was the pressure at the tested shortening velocity and the second the pressure at zero shortening velocity, or the isovolumetric pressure. To these values the Hill equation (1) was fitted, resulting in an estimate for $\mathrm{v}_{\max }$ of the tested bladder at the used level of stimulation. The derivation of $v_{\max }$ from pressure values measured at imposed shortening velocities permitted the use of a sample frequency as low as $10 \mathrm{~Hz}$. The limited number of flow-rate settings of the infusion pump made it necessary to do the measurements at different volumes. This procedure is justified if $v_{\max }$ is volume independent. Theoretically the maximum shortening velocity of muscle is independent of the number of interacting cross-bridges and therefore of the stretched length. ${ }^{6}$ The volume independence of $v_{\max }$ was previously observed in vivo ${ }^{3}$ and was now confirmed in vitro by analysis of the volume dependence of the algebraically derived $v_{\max }$ values (fig. 5). The independence of $v_{\max }$ on bladder volume in the range studied permitted expression of $v_{\max }$ in terms of $\mathrm{cm}$. per second instead of muscle lengths per second; that is, no reference to the volume at which isometric force is maximal was needed. In the derivation of the (active) pressures $\mathrm{P}(\mathrm{v})$ during shortening and $\mathrm{P}_{\text {isv1 } 1}$ during the subsequent isovolumetric part of the contraction, the pressure after the stop-flow contraction had subsided was taken as the passive pressure. This method implies that viscoelastic relaxation during shortening was disregarded, resulting in an underestimation of both the $P(v)$ and the $P_{\text {isv } 1}$ values. Griffiths et al. showed that the viscoelastic effect is small compared with active forces (pressures). ${ }^{7}$ Therefore, the neglect of this effect probably affected the $\mathrm{v}_{\max }$ values only marginally.

Electrical stimulation was used to induce a bladder contraction. Previous work, performed at a bladder volume of 2.8 ml., showed that the stimulation parameters $10 \mathrm{~V}, 200 \mathrm{~Hz}$, 2.5 msec. resulted in optimum stimulation, that is, isovolumetric pressure was maximal, while at the setting $10 \mathrm{~V}, 25$ $\mathrm{Hz}, 20$ msec., pressure was $58 \%$ of maximum. ${ }^{2}$ The $\mathrm{v}_{\max }$ values found at these settings in the present study, $3.75 \pm$ 0.08 and $3.00 \pm 0.22 \mathrm{~cm} . / \mathrm{sec}$., were significantly different as well. It can be concluded that, in our model, both parameters characterizing bladder contractility depended on the intensity of stimulation. The origin of this dependence cannot unequivocally be deduced from our experiments. Possibly the bladder is not uniformly stimulated at a submaximal intensity, implying that parts of the bladder do not contribute to contraction, despite the fact that the geometrical conditions were identical at both stimulation intensities used. In vivo, submaximal neurogenic stimulation might correspond to a situation in which neurotransmitters are not available to a sufficient degree to initiate a uniform contraction in the bladder.

Decay of pressure during stimulation. In the derivation of $\mathrm{v}_{\max }$ the pressure $\mathrm{P}(\mathrm{v})$ at the moment flow stopped was compared with the maximum pressure $P_{\text {isv1 }}$ in the isovolumetric part of the same contraction. These two pressure values were separated in time by about 2 seconds. In the subsequently measured completely isovolumetric contractions, it was observed that, at small bladder volumes, pressure decreased quickly after a maximum had been reached, even while stimulation continued. Because the same phe- 
nomenon may have occurred in the stop-flow contractions where it could not be observed, it was carefully studied to evaluate the degree to which it might have affected the present results. This was done by examining the ratio $\mathrm{P}(6.5) /$ $P_{\text {isv2 }}$, the quotient of the active pressure at the end of the 6.5 second stimulation period and the maximum active pressure attained in this period. Figure 7, B shows that, for both stimulation frequencies, a cut-off circumference, above which pressure remained fairly constant in the time period studied, could be identified. A decay of pressure after the maximum was described earlier in the pig and rabbit bladder, ${ }^{7,8}$ although pressure decreased much more slowly in these bladders. The decrease in pressure while stimulation continues was previously shown to be volume dependent in the rabbit bladder. ${ }^{9}$ It is of interest to realize that the two cut-off circumferences for the two stimulation frequencies were not significantly different. In addition, the degree of decrease in pressure was similar for both stimulation frequencies when bladder circumference was smaller than the cut-off circumference. These findings imply that the observed stimulation intensity dependence of $v_{\max }$ was not biased by a possible decrease in pressure. The independence of $v_{\max }$ of bladder volume (fig. 5) suggests that the $v_{\max }$ values themselves were not biased by such a decrease either.

Effect of preceding shortening on isovolumetric pressure. The two isovolumetric pressure values in each measurement were obtained at exactly the same volume. It was found that these values were considerably different. No dependence of the ratio of these values on bladder volume or stimulation intensity was seen. This suggests that the phenomenon is not due to macroscopic effects of bladder stimulation, but is related to muscle shortening itself. It is known that striated muscle fibers generate less isometric force immediately after active shortening to a certain length compared with an isometric contraction at the same length without preceding shortening. ${ }^{10}$ This shortening-induced weakening of contraction has been called "shortening-induced deactivation". The phenomenon is ascribed to a reduced affinity of the binding sites for calcium on the thin filaments. ${ }^{10}$ Morphologic evidence for deactivation at short lengths was first reported by Taylor and Rüdel. ${ }^{11}$ Our data suggest that shortening-induced deactivation also occurs in the smooth muscle of the guinea pig urinary bladder. As the pressure values measured during shortening in the present experiments should be related to isovolumetric values at the same state of activation, the time delay by which these two pressures are measured should be minimized. Therefore the lower value of $P_{\text {isv1 } 1}$, rather than $P_{\text {isv2 }}$, was used in the derivation of $v_{\max }$. The effect of shortening-induced deactivation measured in the guinea pig bladder, a reduction in isovolumetric pressure of $33 \pm 12 \%$, was much larger than the reduction in isometric force of 4.0 to $7.5 \%$ measured by Edman in striated muscle fibers. ${ }^{10}$ The difference cannot be explained by a possible decay in pressure in the stop-flow contraction, because it was also present at bladder volumes larger than $3.5 \mathrm{ml}$., where isovolumetric pressure remained nearly constant after the maximum (fig. 4, A). The deactivation being shortening-induced, a negative regression coefficient of the ratio $P_{\text {isv }} / \mathrm{P}_{\text {isv2 }}$ on the shortening velocity was expected. Such a relationship was not found. Edman showed that the degree of force reduction was not markedly affected by shortening velocities in the range of $v_{\max }$ to $1 / 5$ of $v_{\max } \cdot{ }^{12}$

The effect of shortening on the isometric force has been demonstrated in whole muscle in situ ${ }^{12}$ and might also exist in the human bladder. The duration of the effect is unknown from our experiments. Unless this period is very short, it has implications for the clinically employed stop-flow test. In this test the patient is asked to interrupt micturition. The isovolumetric pressure occurring afterwards is used to assess bladder contractility. Deactivation would underestimate it.

\section{REFERENCES}

1. Groen, J., van Asselt, E., van Mastrigt, R., Bosch, R. and van Koeveringe, G. A.: Comparison of detrusor contractility of guinea pig bladders in situ and strips from these in vitro. J. Urol., 150: 1002, 1993.

2. Groen, J., van Mastrigt, R. and Bosch, R.: Contractility of the guinea pig bladder measured in situ and in vitro. Neurourol. Urodyn., 13: 587, 1994.

3. Groen, J., van Mastrigt, R., van Asselt, E., van Koeveringe, G. A. and Bosch, R.: Contractility parameters of the guinea pig bladder in situ: similarity to human bladder contractility. J. Urol., 151: 1405, 1994.

4. Griffiths, D. J. and van Mastrigt, R.: The routine assessment of detrusor contraction strength. Neurourol. Urodyn., 4: 77, 1985.

5. van Mastrigt, R. and Griffiths, D. J.: Contractility of the urinary bladder. Urol. Int., 34: 410, 1979.

6. Huxley, A. F.: Muscle structure and theories of contraction. Prog. Biophys. Biophys. Chem., 7: 255, 1957.

7. Griffiths, D. J., van Mastrigt, R., van Duyl, W. A. and Coolsaet, B. L. R. A.: Active mechanical properties of the smooth muscle of the urinary bladder. Med. Biol. Eng. Comput., 17:281, 1979.

8. Levin, R. M., Ruggieri, M. R., Gill, H. S., Haugaard, N. and Wein, A. J.: Studies on the biphasic nature of urinary bladder contraction and function. Neurourol. Urodyn., 6: 339, 1987.

9. Kaplan, S. A., Blaivas, J. G., Brown, W. C. and Levin, R. M. Parameters of detrusor contractility. I. The effects of electrical stimulation, hysteresis, and bladder volume in an in vitro whole rabbit bladder model. Neurourol. Urodyn., 10: 53, 1991.

10. Edman, K. A. P.: Depression of mechanical performance by active shortening during twitch and tetanus of vertebrate muscle fibers. Acta Physiol. Scand., 109: 15, 1980.

11. Taylor, S. R. and Rüdel, R.: Striated muscle fibers: inactivation of contraction induced by shortening. Science, 167: 882, 1970.

12. Edman, K. A. P.: Mechanical deactivation induced by active shortening in isolated muscle fibres of the frog. J. Physiol., 246: 255, 1975. 